\title{
GAIA Survey of Galactic Eclipsing Binaries
}

\author{
Tomaž Zwitter \\ University of Ljubljana, Department of Physics, Jadranska 19, 1000 \\ Ljubljana, Slovenia
}

\begin{abstract}
The general importance and capabilities of observations of eclipsing binaries by the forthcoming ESA mission GAIA are discussed. Availability of spectroscopic observations and a large number of photometric bands on board will make it possible to reliably determine physical parameters for $\sim 10^{5}$ binary stars. It is stressed that current methods of object by object analysis will have to be modified and included in an automatic analysis pipeline.
\end{abstract}

\section{Introduction}

GAIA is the approved Cornerstone 6 mission of the European Space Agency. Its main goal is to observe up to a billion stars in our Galaxy and obtain their astrometric positions on a micro-arcsec level, multi-band photometry in 15 different optical and near-IR bands, as well as spectroscopic observations within the $250 \AA$ interval around the CaII IR triplet. Perryman et al. (2001), ESA-SCI(2000)4 and Munari $(1999,2002)$ are useful introductions to the general properties of the mission, its overall astrophysical importance and diagnostic capabilities of the spectral window chosen for the spectrograph. Munari (page 25) discusses the potential of GAIA to observe peculiar stars. Here we focus on galactic eclipsing binaries which are rapidly becobing one of the areas where the harvest of GAIA's results will be the richest.

In the next section we discuss general importance and capabilities of observations of eclipsing binaries by GAIA. Next the mode in which spectroscopic observations are obtained is briefly discussed, together with sources of noise, intensity of the background and the possibility for spectral tracing overlaps. All this leads to the estimates on expected radial velocity accuracy as a function of magnitude and spectral type of the target. Finally we present results from observations of real binary stars in the GAIA-like mode and so demonstrate GAIA's accuracy in determination of basic stellar parameters. We conclude with some remarks on automatic reduction of the gigantic database of GAIA's observations of galactic eclipsing binaries.

\section{Importance and capabilities of binary star observations}

We shall illustrate the capabilities of observations of binary stars for two cases: stars brighter than $V=15$, and stars brighter than $V=17$. For the former class the errors on single-star parameters obtained from a combination of astrometry, 


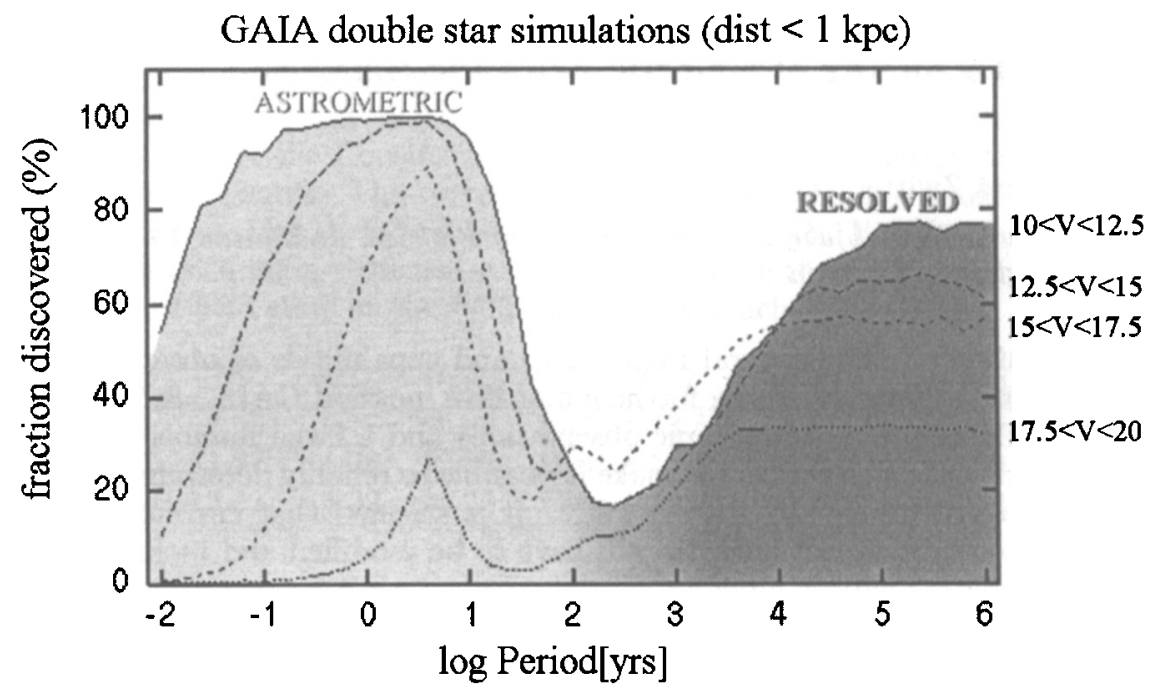

Figure 1. Fraction of astrometric and resolved binary stars discovered by GAIA as a function of orbital period and for different magnitude ranges. Simulations were done for a distance limited sample $(d<1 \mathrm{kpc})$. Adapted from ESA-SCI $(2000) 4$.

photometry and spectroscopy will be less than $50 \mathrm{~K}$ for the effective temperature, less than 0.1 for gravity, less than 0.2 for metallicity and less than $10 \%$ for distance (for distances within $10 \mathrm{kpc}$ ) (see ESA-SCI(2000)4). These values could be checked by observations of some $4 \times 10^{5}$ eclipsing binaries with $V<15$. Of these some $\sim 10^{5}$ will be double-lined. It is not too optimistic to expect that one can obtain a few ten-thousand excellent orbital solutions with secure determination of stellar parameters, a huge increase over the current number of reliably solved eclipsing binaries. Apart from measurement of stellar parameters that cannot be independently determined for single stars (mass and to some extent radius) GAIA's orbital solutions of bright eclipsing binaries will have the potential to determine their distances even for objects much farther than $10 \mathrm{kpc}$, the base-lined $10 \%$ accuracy limit for astrometry.

For fainter targets $(V<17$, distance $<10 \mathrm{kpc})$ GAIA will provide observations of $\sim 5 \times 10^{7}$ stars. It is important to note that all these stars will be observed in exactly the same way. Some of the stars will be single, but others will be binaries with orbital periods from hours to hundreds of years. The obvious question one can address with this database is how binarity influences stellar evolution. Eclipsing binaries will be observed and discovered also at fainter magnitudes. Good quality light curves could be obtained but spectroscopic observations will be of limited accuracy. Current galactic models (see ESA-SCI(2000)4) predict discovery of $\sim 7 \times 10^{6}$ eclipsing binaries at $V<20$. 
GAIA will survey a wide range of binary star separations and orbital periods (Fig. 1). At the longest orbital periods ( $>100 \mathrm{yrs}$ ) the components will be resolved on the sky and their binary nature could be judged from their equal parallax, proper motion and a similar radial velocity. Closer binaries, down to orbital periods of days, will be discovered by the curved, non-linear proper motion path. Finally, spectroscopy will be useful to discover even shorter period binaries, not plotted in Fig. 1. As seen from the Figure the fraction of discovered binaries is significant, thus GAIA will be able to study the binarity phenomenon for the whole range of orbital periods.

\section{Performance of the spectrograph}

The spectrograph aboard GAIA will have a square primary mirror with the surface area of $0.5 \times 0.5 \mathrm{~m}^{2}$ and the field of view of $1.65^{\circ} \times 1.6^{\circ}$. It is a slitless spectrograph with stars drifting across the focal plane at the rate of $60^{\prime \prime} / \mathrm{s}$. This gives a fixed exposure time of 99 seconds for each transit of the star across the focal plane. Because of a precessional motion of the spin axis of the satellite the whole celestial sphere will be covered, and each position on the sky will be observed on average 100-times during a 5-year mission.

The spectral interval is base-lined to $8490-8750 \AA$. It includes Paschen lines P13-P16, the Ca II IR triplet as well as numerous N I, Fe I, Ti I, Cr I, Si I, Ni I, S II, $\mathrm{MgI}$ and MnI lines (Munari 2002). The resolution will be most likely between 10000 and 15000 .

The performance of the spectrograph will be limited by shot noise, RON, zodiacal background and - to some extent - spectral tracing overlaps. Zodiacal background was discussed by Zwitter (2002). Overlapped spectral tracings will not present a significant problem in terms of spectral line blending, as stars' angular separation on the sky guarantees that lines generally do not overlap. Moreover the scanning law is such that two spectral tracings overlapping on one transit will be recorded separately on the next occasion of observation of the same field. The main contribution of spectral overlaps will thus be an increase in the effective background. This will become significant for stars fainter than $V \sim$ 17 (except in the galactic plane where stellar overlaps will be more common).

Fig. 2 summarizes the expected radial velocity accuracy as a function of magnitude and spectral type. The zodiacal background of $V=21.5 / \operatorname{arcsec}^{2}$ was assumed, which is typical for an object on the ecliptic plane. This figure is the same as Fig. 4 of $\mathrm{Zwitter}$ (2002), but calculated for updated parameters of the spectrograph mentioned above. At the bright magnitude end higher resolution always gives better accuracy. For the faintest targets the accuracy achieved by the highest resolution $(0.25 \AA / \mathrm{pix})$ is degraded due to the fact that the Zodiacal background per wavelength bin does not depend on resolution, while the stellar signal is more dispersed at higher resolutions. Similar results were obtained also by observations of real stars (Munari, Agnolin, \& Tomasella 2001).

\section{Observations and analysis of galactic eclipsing binaries}

Most binaries observed by GAIA will contain dwarfs of type G or K. Radial velocity accuracies mentioned above demonstrate that the GAIA's spectrograph 

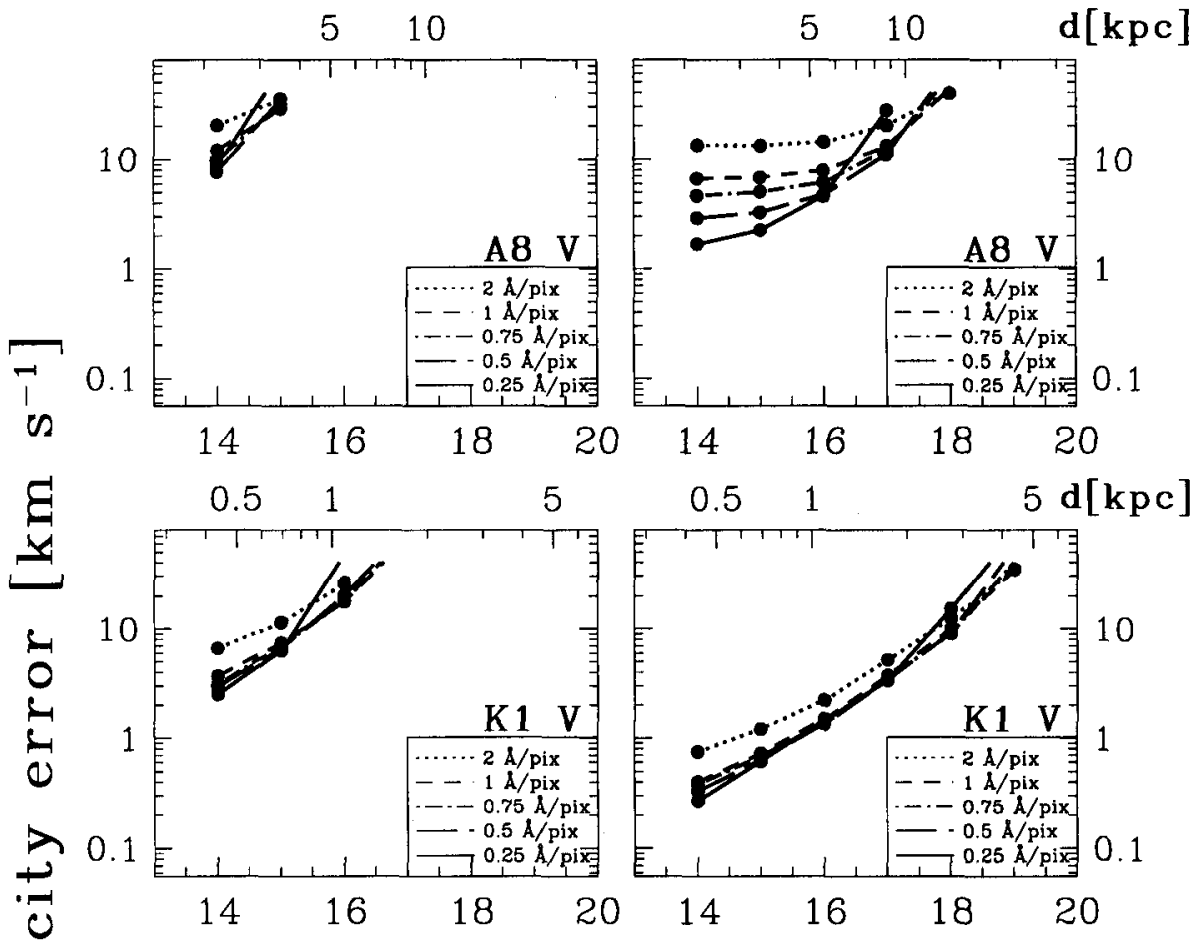

$\frac{0}{0}$
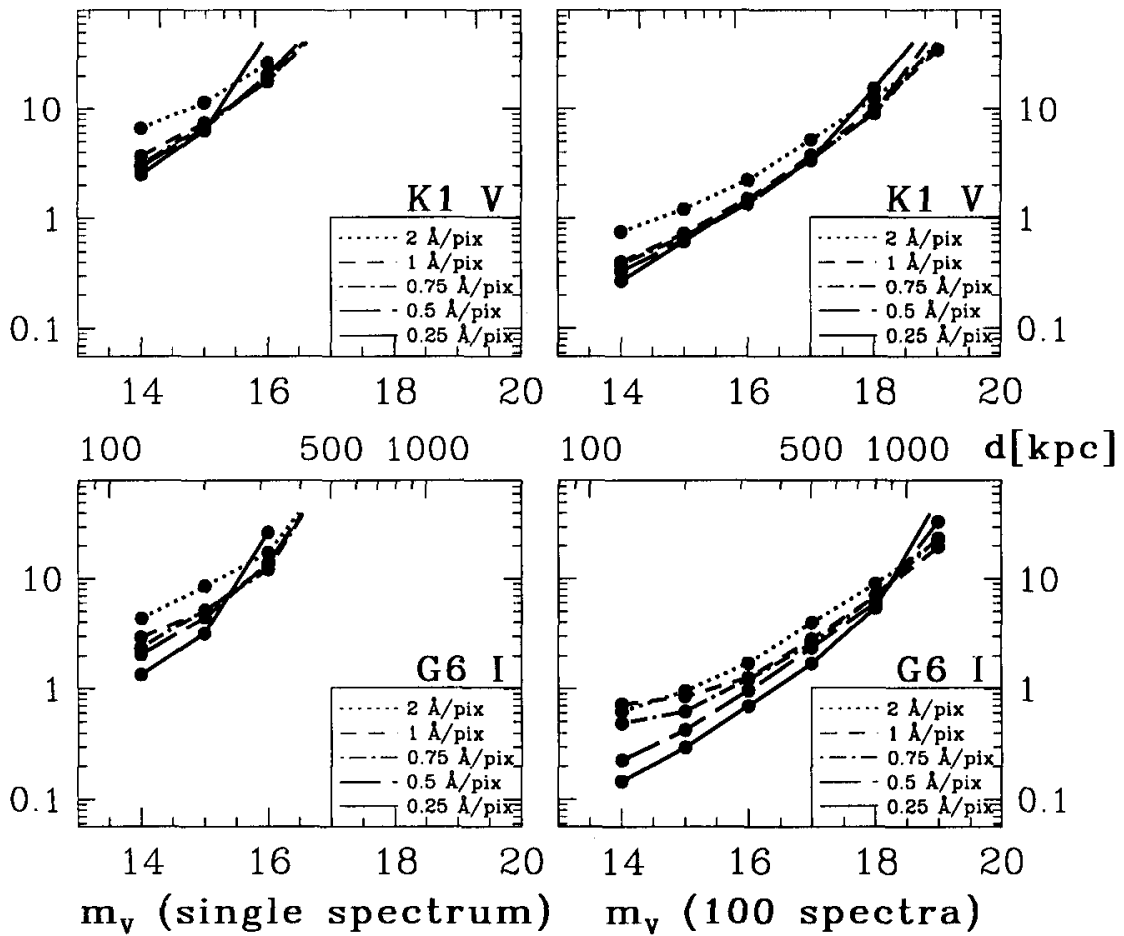

Figure 2. Average radial velocity determination error as a function of stellar magnitude, spectral type and the used spectral dispersion (assuming 2-pixels per resolution element). Top labels at each graph quote a representative distance if no interstellar absorption is present. Left column is for single transit spectra, while the right is for a missionaveraged spectrum (100 transits). 


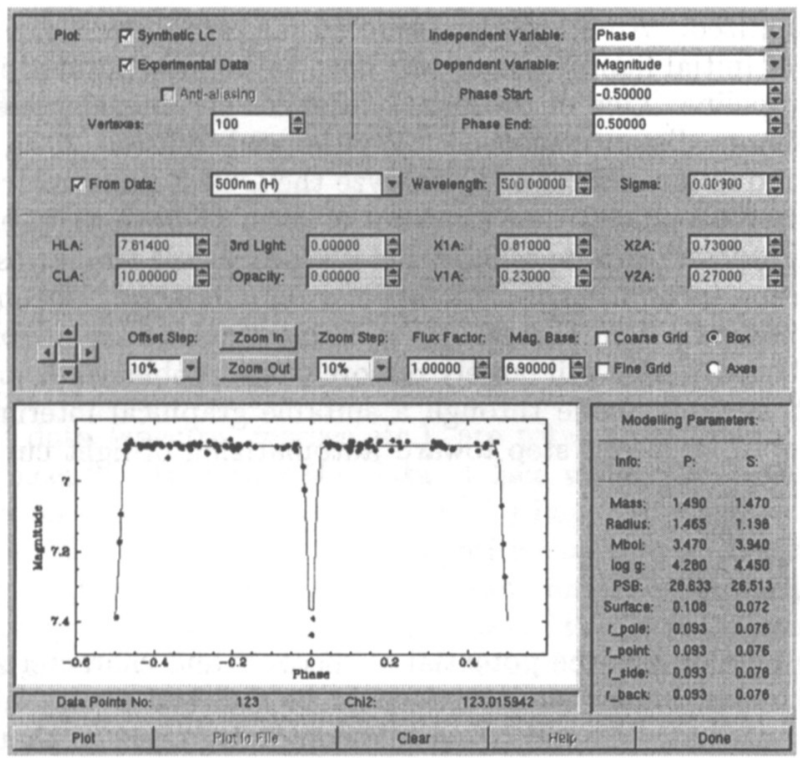

Figure 3. Graphical user interface to run the Wilson-Devinney program (A. Prša 2002, private communication).

will be able to secure useful radial velocity curves for binaries down to $V \sim 17$. GAIA will also obtain $\sim 100$ photometric observations of each binary in each of its 11 narrow- and 4 broad-band photometric filters. These can be used to determine stellar temperatures, gravities as well as provide some information on the metallicity. Typical errors for single stars will be $125 \mathrm{~K}$ in $T_{e f f}, 0.25$ in $\log g$ and 0.4 in $\left[Z / Z_{o}\right](\mathrm{ESA}-\mathrm{SCI}(2000) 4)$. These values can be further improved by spectra fitting.

For eclipsing binaries the light curve and spectra analysis can improve the accuracy further. Munari et al. (2001) published spectroscopic observations in the GAIA spectral window of three eclipsing binaries discovered by the Hipparcos satellite. Only three Hipparcos photometric bands with very limited S/N instead of the 14 bands previewed for GAIA were used in light curve analysis. Still the results have very acceptable error-bars: $\sim 2 \%$ in masses, $\sim 100 \mathrm{~K}$ in temperatures, $\sim 0.1$ in $\log g$, and $\sim 7 \%$ in distance. These results are encouraging and demonstrate that GAIA data will be suitable to obtain accurate binary solutions. Reliable information on temperature, supplemented by actual fitting of the template spectra at each of the $\sim 100$ transits, opens the possibility to detect and analyze magnetic spots on the surfaces of eclipsing binaries which should be quite common for cool dwarfs. Such variability was also detected in yet unpublished eclipsing binaries discovered by Hipparcos that we have been observing in Asiago in the past 3 years.

As mentioned above the number of eclipsing binaries discovered by GAIA will reach hundred-thousands. This means that the analysis will have to be 
fully automatized. All steps that were so far performed by astronomers, like the choice of initial fitting values, will have to be incorporated in an integrated reduction pipeline. One of the possibilities for the initial value determination is the minimum distance method. Wyithe and Wilson $(2001,2002)$ used an automatic reduction technique to analyze the OGLE photometric database. As expected they concluded that the sum of radii of both stars and the ratio of surface brightnesses can be reliably determined, while the ratios of radii, ratios of luminosities, inclination and eccentricity remain largely unconstrained by the OGLE photometric light curves. It should be stressed that the presence of spectroscopy aboard GAIA will largely remove these problems. A program running the Wilson-Devinney code through a suitable graphical interface (Fig. 3) has been developed as a first step toward automatizing of light curve and spectral analysis.

\section{Conclusions}

The GAIA mission has the potential to discover and characterize thousands of new peculiar and binary stars that could be followed up by additional observations by automatic ground based telescopes. It is crucial that suitable codes are developed for a correct recognition of eclipsing and non-eclipsing binaries on a central reduction pipeline. Once recognized the light curves and spectra will need to be analyzed with an automatic and reliable automatic procedures.

The unique potential of GAIA among the forthcoming space missions is the availability of spectroscopy on board and an unprecedented number of targets that are to be observed. The size of the database at the end of the mission will exceed 10 TB. However, as correctly pointed out by Peter Eggleton, more data can mean less understanding. To avoid this it is very important to think now of the new era when even peculiar binaries will be observed in large numbers.

Acknowledgments. This work has been supported by a grant from the Slovenian Ministry of Education, Science and Sport.

\section{References}

Munari, U. 1999, in Proceedings of the ESA Leiden Workshop on GAIA, ed. V. Strayzis, Balt. Astron., 8, 73

Munari, U. 2002, in GAIA, an European Space Project, ed. O. Bienaymé \& C. Turon (Paris: Editions de Physique), 39

Munari, U., Agnolin, P., Tomasella, L., 2001, Baltic Astronomy, 10, 613

Munari, U., et al. 2001, A\&A, 378, 477

Perryman, M. A. C., et al. 2001, A\&A, 369, 339

Wyithe, J. S. B., Wilson, R. E. 2001, ApJ, 559, 260

Wyithe, J. S. B., Wilson, R. E. 2002, ApJ, 571, 293

Zwitter, T. 2002, A\&A, 386, 748 